\section{Microbiological evaluation of hot beverages dispensed by vending machines from the Army barracks of Brigata Meccanizzata Aosta located in Messina}

\author{
Attilio Longo, ${ }^{1}$ Alessandro Giuffrida, ${ }^{2}$ \\ Filippo Giarratana, ${ }^{2}$ Chiara Beninati, ${ }^{2}$ \\ Daniele Muscolino, ${ }^{2}$ Antonio Panebianco ${ }^{2}$ \\ ${ }^{1}$ Esercito Italiano, Messina; ${ }^{2}$ Dipartimento \\ di Scienze Veterinarie, Università degli \\ Studi di Messina, Messina, Italy
}

\section{Abstract}

The aim of the present study was to evaluate the microbiological quality of hot beverages dispensed by vending machines (VMs). The study was carried out on 203 samples from 15 VMs located in 5 Army barracks in Messina. The samples included: water used for preparation of beverages, swab of water tank, swab of blender machine, chocolate powder, milk powder, cappuccino and chocolate drink (29 samples for each types). All samples were examined for total bacterial count (TBC), coliforms, Escherichia coli, enterococci, Pseudomonas aeruginosa, Staphylococcus aureus, Clostridium perfringens, Aeromonas spp., Salmonella spp. and Listeria monocytogenes. For the water samples the colony count (CC) at $22^{\circ} \mathrm{C}$ and at $37^{\circ} \mathrm{C}$ was made. The average values of $\mathrm{CC}$ at $22^{\circ} \mathrm{C}$ and at $37^{\circ} \mathrm{C}$ were of $10.86 \times 10^{2} \pm 8.72 \times 10^{2}$ $\mathrm{CFU} / \mathrm{mL}$ and of $21.72 \times 10^{2} \pm 16.44 \times 10^{2} \mathrm{CFU} / \mathrm{mL}$ respectively. $P$. aeruginosa, coliform bacteria, $S$. aureus, E. coli and molds were detected from water. The TBC ranged from $176 \mathrm{CFU} / \mathrm{g}$ $( \pm 275.2)$ for chocolate powder to $294.8 \pm 69.4$ $\mathrm{CFU} / \mathrm{g}$ for milk powder. $S$. aureus and molds were isolated from milk powder, while coliforms, $E$. coli and $S$. aureus were observed in chocolate powder. The average TBC for hot beverages ranged from $34.32 \times 10^{3} \pm 97.77 \times 10^{3}$ $\mathrm{CFU} / \mathrm{mL}$ for cappuccino to $36.59 \times 10^{3} \pm 10.47$ $\times 10^{4} \mathrm{CFU} / \mathrm{mL}$ for chocolate drink. Coliforms, $E$. coli, enterococci and molds were detected from cappuccino, while enterococci and molds were observed in chocolate drink. The microbiological characteristics of the water and powders, hygiene, and the periodic cleaning of machines, influenced the microbiological quality of the hot beverages dispensed by VMs.

\section{Introduzione}

Comparsi per la prima volta in Italia all'ini- zio negli anni Cinquanta, i distributori automatici (DA) furono importati dagli Stati Uniti per la distribuzione di bibite imbottigliate e solo dopo un decennio furono messe a punto le prime macchine in grado di erogare il caffè espresso. Nel corso degli anni, grazie alla notevole praticità d'uso unita alle diverse abitudini alimentari, si è osservato un crescente aumento di tali apparecchiature nei posti di lavoro, nelle scuole, nelle mense, negli ospedali, ecc., unitamente a una notevole diversificazione delle apparecchiature e dei prodotti erogabili. I DA oggi sono, infatti, capaci di erogare una grande varietà di bevande, confezionate e non, e di alimenti solidi, che vanno dallo snack mantenuto a temperatura di refrigerazione sino alla pizza appena sfornata. La maggior parte di tali apparecchiature è costituita comunque da dispensatori di caffè espresso e di bevande solubili (the, latte, cioccolata calda, ecc.) e possono essere inoltre distinte in automatiche e semiautomatiche. Queste ultime richiedono, rispetto alle automatiche, una maggiore interazione con l'utilizzatore, il quale deve scegliere la cialda di proprio gradimento, inserirla nell'apposito spazio e predisporre il bicchiere in attesa dell'erogazione della bibita, inoltre deve provvedere a ricaricare il serbatoio dell'acqua. Nei distributori automatici, invece, le bevande sono prodotte a partire da caffè in grani 0 da polveri solubili, conservate separatamente in appositi contenitori, e miscelati con l'acqua solo al momento della selezione. La bevanda viene infine raccolta in bicchiere di plastica alimentare usa e getta, e come tale è servita all'utente. Dalla letteratura scientifica, in verità non molto numerosa (Hall, 2008; Hunter e Burge 1986; Vallone e Bonomi, 2011; White et al., 2010), sulla qualità microbiologica delle bevande erogate da distributori automatici sembra emergere una notevole variabilità condizionata, principalmente, dalla qualità microbiologica dell'acqua impiegata e delle polveri che entrano nella composizione delle bevande nonché dall'igiene e dalla pulizia dei DA. Il grado di contaminazione delle bevande erogate potrebbe essere inoltre influenzato dall'intervallo di tempo che intercorre tra due pulizie successive, la tipologia di disinfettante impiegato, dal tipo più o meno complesso di apparecchiatura, nonché dalla varietà di bevande distribuite, dall'acidità e dal dosaggio delle materie prime. Scopo della presente ricerca è stato quello di contribuire alla conoscenza dei fattori che influiscono sulla qualità microbiologica di bevande calde erogate da dispensatori automatici.

\section{Materiali e Metodi}

L'attività di campionamento è stata condotta nel dicembre del 2010 su un totale di 15 DA di
Correspondence: Filippo Giarratana, Dipartimento di Sanità Pubblica Veterinaria, Università degli Studi di Messina, viale Annunziata, 98168 Messina, Italy.

Tel. +39.90.3503768 - Fax: +39.90.3503937.

E-mail: fgiarratana@unime.it

Key words: Vending machines, Hot drink, Water, Microbiology.

Conflict of interests: the authors declare no potential conflict of interests.

Received for publication: 15 January 2013.

Revision received: 22 February 2013.

Accepted for publication: 23 February 2013.

This work is licensed under a Creative Commons Attribution 3.0 License (by-nc 3.0)

(C) Copyright A. Longo et al., 2013

Licensee PAGEPress, Italy

Italian Journal of Food Safety 2013; 2:e5

doi:10.4081/ijfs.2013.e5

bevande presenti presso vari reparti delle 5 caserme della Brigata Meccanizzata Aosta dell'Esercito Italiano della città di Messina.

I suddetti distributori appartenevano tutti alla medesima ditta. Per ogni distributore si procedeva al prelievo, in contenitori sterili, dell'acqua utilizzata per la reidratazione delle bevande contenuta al loro interno in serbatoi di plastica. Sulle superfici interne di tali serbatoi e sul tubo del sistema di aspirazione dell'acqua veniva, inoltre, effettuato un tampone (denominato tampone acqua). Seguiva il prelievo delle polveri di latte e cioccolato e delle rispettive bevande reidratate indicate come cappuccino e bevanda al cioccolato. Infine, si effettuava un tampone sulle superfici interne del miscelatore e del beccuccio dell'erogatore che erano comuni ad entrambe le bevande prelevate (denominato tampone miscelatore).

Le medesime procedure di campionamento venivano ripetute a distanza di una settimana, al fine di permettere alla ditta responsabile di effettuare eventuali operazioni di pulizia ordinaria/straordinaria, ottenendo complessivamente 203 campioni. Nel corso del secondo campionamento si rilevava la temperatura di erogazione delle bevande e dell'acqua di lavaggio, al fine di stimare rispettivamente l'eventuale influenza della temperatura sulla qualità microbiologica delle bevande e il grado di efficienza del sistema di lavaggio.

Tutti i campioni venivano immediatamente refrigerati e trasportati presso i laboratori della Sezione di Ispezione degli Alimenti di Origine Animale del Dipartimento di Scienze Veterinarie di Messina.

In base alla tipologia di campione si procedeva, secondo le metodiche ufficiali o le comuni tecniche di laboratorio, alla determinazione 
quantitativa e/o alla ricerca dei seguenti parametri: i) Campioni acqua: conteggio delle colonie su agar a $22^{\circ} \mathrm{C}$ e a $37^{\circ} \mathrm{C}$ (ISS A 004A rev. 00); determinazione di Escherichia coli (ISS A 001B rev. 00); determinazione dei batteri coliformi a $37^{\circ} \mathrm{C}$ (ISS A $006 \mathrm{C}$ rev. 00); determinazione degli enterococchi (ISS A 002 A rev. 00); determinazione di Pseudomonas aeruginosa (ISS A 003A rev. 00); determinazione di Clostridium perfringens (ISS A 005A rev. 00); determinazione di Staphylococcus aureus (ISS A 018A rev. 00); determinazione di Aeromonas spp. (ISS A 014 rev. 00); determinazione di muffe (ISS A 016C rev.00); ricerca di Salmonella spp. [filtrazione su membrana di 1 L di acqua e successivo impiego della UNI EN ISO 6579:2008 (ISO, 2008)] e di Listeria monocytogenes [filtrazione su membrana di $1 \mathrm{~L} \mathrm{di}$ acqua e successivo impiego della UNI EN ISO 11290-1:2005 (IS0, 2005)]. ii) Campioni polveri e bevande: conteggio della carica batterica totale (CBT) (PCA a $30^{\circ} \mathrm{C}$ per $72 \mathrm{~h}$ ); determinazione di $E$. coli (UNI EN ISO 16649-2:2001) (ISO, 2001); determinazione dei batteri coliformi a $37^{\circ} \mathrm{C}$ (UNI EN ISO 4832:2006) (ISO, 2006); determinazione di enterococchi (Slanetz and Bartley medium a $37^{\circ} \mathrm{C}$ per $48 \mathrm{~h}$ ); determinazione di $P$. aeruginosa (Pseudomonas agar base con $\mathrm{CN}$ supplement a $30^{\circ} \mathrm{C}$ per $24 \mathrm{~h}$ ); determinazione di $C$. perfringens (ISTISAN 96-35); determinazione di $S$. aureus (UNI EN ISO 6888-1:1999) (ISO, 1999); determinazione di Aeromonas spp. (Aeromonas agar base Ryan a $30^{\circ} \mathrm{C}$ per $24 \mathrm{~h}$ ); determinazione di muffe (Malt Extract Agar incubate a $25^{\circ} \mathrm{C}$ per $5 \mathrm{gg}$ ); ricerca di Salmonella spp. (ISO, 2008) e di L. monocytogenes (ISO, 2005). iii) Campioni tamponi: su questi si effettuata la ricerca qualitativa di $E$. coli e coliformi (ChromoCult Coliform agar a $37^{\circ} \mathrm{C}$ per $24 \mathrm{~h}$ ); enterococchi (Slanetz and Bartley medium a $37^{\circ} \mathrm{C}$ per $24 \mathrm{~h}$ ); $P$. aeruginosa (Pseudomonas agar base a $30^{\circ} \mathrm{C}$ per $24 \mathrm{~h}$ ); $C$. perfringens (Perfringens agar base TSC a $37^{\circ} \mathrm{C}$ per $20 \mathrm{~h}$ ); $S$. aureus (Baird-Parker agar base con egg yolk tellurite emulsion a $37^{\circ} \mathrm{C}$ per $24 \mathrm{~h}$ ); Aeromonas spp. (Aeromonas agar base Ryan a $30^{\circ} \mathrm{C}$ per 24 h), muffe (Malt extract agar a $25^{\circ} \mathrm{C}$ per $5 \mathrm{gg}$ ); Salmonella spp. (ISO, 2008) e L. monocytogenes (IS0, 2005).

La valutazione di eventuali correlazioni tra temperatura di erogazione delle bevande e relativa CBT veniva effettuata mediante regressione lineare.

\section{Risultati}

Nel corso del primo campionamento, già durante le fasi di prelievo, emergevano diverse criticità, sullo stato di igiene e pulizia dei DA esaminati. Tali criticità erano riconducibili alla mancanza di un sistema di chiusura erme- tica in alcuni serbatoi dell'acqua, atti ad impedire l'ingresso di insetti, ma soprattutto alla presenza, all'interno del sistema di aspirazione e di circolazione dell'acqua, di uno spesso biofilm mucillaginoso brunastro. Altra criticità era legata alla gestione dei fondi di caffè, in quanto questi venivano raccolti in contenitori di plastica aperti all'interno degli stessi distributori automatici. La loro permanenza, anche per diversi giorni, all'interno dei DA, condizionata dai tempi intercorrenti tra una ricarica e l'altra dell'apparecchiatura, catalizzava costantemente lo sviluppo di muffe. Malgrado quanto segnalato alla ditta, nell'ambito del secondo campionamento non si evidenziava un miglioramento significativo delle condizioni igieniche dei DA, peraltro, aggravate dal riscontro di un topo morto in uno di essi (e quindi escluso dalla serie di prelievi) che testimonierebbe una criticità anche di tipo progettuale dei DA. Al riguardo, ricordiamo che nel manuale di corretta prassi igienica dell'Associazione Italiana Distribuzione Automatica (Dragoni e Bonomi, 2007), alla luce del regolamento 852 della Commissione Europea (2004) si sottolinea che i DA devono per quanto ragionevolmente possibile, essere situati, progettati e costruiti, nonché mantenuti puliti e sottoposti a regolare manutenzione in modo tale da evitare rischi di contaminazione, in particolare da parte di animali e di animali infestanti.

Dal punto di vista batteriologico, come riportato nella Tabella 1, i 29 campioni di acqua analizzati presentavano valori estremamente variabili di carica batterica a $22^{\circ} \mathrm{C}$ e a $37^{\circ} \mathrm{C}$ con valori medi pari rispettivamente a $10,86 \mathrm{x}$ $10^{2} \pm 8,72$ x $10^{2}$ unità formanti colonie (UFC)/mL e a $21,72 \times 10^{2} \pm 16,44 \times 10^{2} \mathrm{UFC} / \mathrm{mL}$. Le muffe si isolavano in ben 27 campioni (93,1\%), seguiti da $P$. aeruginosa in 18 $(62,1 \%)$, dai coliformi in $10(34,5 \%)$, da $E$. coli in $9(31 \%)$ e infine da $S$. aureus in un solo cam- pione $(3,4 \%)$. Sempre assenti risultavano $C$. perfringens, Aeromonas spp., Salmonella spp. e L. monocytogenes.

Dai 29 campioni denominati tampone acqua si rilevava la presenza di coliformi in 10 campioni $(34,5 \%)$, di $E$. coli in $4(13,8 \%)$, di enterococchi in $6(20,7 \%)$, di $P$. aeruginosa in 7 $(24,1 \%)$, di Aeromonas spp. in $1(4,4 \%)$ e di muffe in $6(20,7 \%)$.

Sui 29 tamponi miscelatore si riscontravano coliformi in 3 campioni $(10,3 \%), E$. coli in 1 (3,4\%), enterococchi in $15(51,7 \%)$, P. aeruginosa in $1(3,4 \%)$, Aeromonas spp. in $1(3,4 \%) \mathrm{e}$ muffe in 9 (31\%). Da entrambi i tamponi (acqua e miscelatore) non si isolavano $C$. perfringens, S. aureus, Salmonella spp. e L. monocytogenes.

La qualità microbiologica delle polveri variava in funzione della tipologia. Il latte in polvere, infatti, presentava valori medi di CBT pari a $294,8 \pm 69,4 \mathrm{UFC} / \mathrm{g}, S$. aureus si isolava in un solo campione $(3,4 \%)$ mentre le muffe in ben 18 campioni (62\%) (Tabella 2). Estremamente variabile la CBT del cioccolato in polvere con valori medi di $176 \pm 275,2 \mathrm{UFC} / \mathrm{g}$; in 7 campioni si isolavano coliformi $(24,1 \%)$, in $4 \mathrm{E}$. coli $(13,8 \%)$ e in un solo campione $S$. aureus $(3,4 \%)$ (Tabella 3$)$.

I 29 campioni della bevanda cappuccino evidenziavano una CBT estremamente variabile con valori medi pari a $34,32 \times 10^{3} \pm 97,77 \times 10^{3}$ UFC/mL. Nel $14 \%$ si riscontravano coliformi, nel $10 \%$ E. coli, nel $28 \%$ enterococchi e nel $69 \%$ muffe (Tabella 2). La temperatura media della bevanda cappuccino, determinata al momento dell'erogazione, era di $67,5 \pm 6,0^{\circ} \mathrm{C}$ con valori variabili da $55^{\circ}$ a $76,8^{\circ} \mathrm{C}$. Anche i 29 campioni della bevanda al cioccolato erano caratterizzati da una notevole variabilità con CBT media di $36,59 \times 10^{3} \pm 10,47 \times 10^{4} \mathrm{UFC} / \mathrm{mL}$ (Tabella 3 ). Nel $17 \%$ e nel $55 \%$ dei campioni si rilevavano, rispettivamente, enterococchi e muffe. La tem-

Tabella 1. Livelli di contaminazioni dell'acqua.

\begin{tabular}{lll} 
Acqua & Campioni positivi (\%) & Media positivi \\
Conteggio colonie a $22^{\circ} \mathrm{C}$ & $29(100 \%)$ & $10,86 \times 10^{2} \pm 8,72 \times 10^{2} \mathrm{UFC} / \mathrm{mL}$ \\
Conteggio colonie a $37^{\circ} \mathrm{C}$ & $29(100 \%)$ & $21,72 \times 10^{2} \pm 16,44 \times 10^{2} \mathrm{UFC} / \mathrm{mL}$ \\
\hline Coliformi a $37^{\circ} \mathrm{C}$ & $10(34,5 \%)$ & $14,1 \pm 11,7 \mathrm{UFC} / 100 \mathrm{~mL}$ \\
E. coli & $9(31 \%)$ & $11,8 \pm 11,9 \mathrm{UFC} / 100 \mathrm{~mL}$ \\
\hline Enterococchi & $0(0 \%)$ & $0 \mathrm{UFC} / 100 \mathrm{~mL}$ \\
P. aeruginosa & $18(62,1 \%)$ & $88,3 \pm 121,8 \mathrm{UFC} / 250 \mathrm{~mL}$ \\
\hline C. perfringens & $0(0 \%)$ & $0 \mathrm{UFC} / 100 \mathrm{~mL}$ \\
S. aureus & $1(3,4 \%)$ & $8 \mathrm{UFC} / 250 \mathrm{~mL}$ \\
\hline Aeromonas spp. & $0(0 \%)$ & $0 \mathrm{UFC} / 100 \mathrm{~mL}$ \\
Muffe & $27(93,1 \%)$ & $10,4 \pm 11,7 \mathrm{UFC} / 100 \mathrm{~mL}$ \\
\hline L. monocytogenes & $0(0 \%)$ & assenza/1 L \\
Salmonella spp. & $0(0 \%)$ & assenza/ $1 \mathrm{~L}$ \\
\hline
\end{tabular}

UFC, unità formanti colonie; E. coli, Escherichia coli; P. aeruginosa, Pseudomonas aeruginosa; C. perfringens, Clostridium perfringens; S. aureus, Staphylococcus aureus; Aeromonas spp., Aeromonas species; L. monocytogenes, Listeria monocytogenes; Salmonella spp., Salmonella species. 
Tabella 2. Livelli di contaminazione del latte in polvere e della relativa bevanda.

$\begin{array}{ccccc}\text { Media positivi UFC/g } & \begin{array}{c}\text { Latte in polvere } \\ \text { Campioni positivi (\%) }\end{array} & \text { Analisi } & \text { Bevanda cappuccino } \\ \text { Media positivi UFC/mL }\end{array}$

UFC, unità formanti colonie; CBT, carica batterica totale.

Tabella 3. Livelli di contaminazione del cioccolato in polvere e della relativa bevanda.

\begin{tabular}{|c|c|c|c|c|}
\hline \multicolumn{2}{|c|}{ Cioccolato in polvere } & \multirow[t]{2}{*}{ Analisi } & \multicolumn{2}{|c|}{ Bevanda al cioccolato } \\
\hline Media positivi UFC/g & Campioni positivi (\%) & & Campioni positivi (\%) & Media positivi UFC/mL \\
\hline $176 \pm 275,2$ & $29(100 \%)$ & $\mathrm{CBT}$ a $32^{\circ} \mathrm{C}$ & $29(100 \%)$ & $36,59 \times 10^{3} \pm 10,47 \times 10^{4}$ \\
\hline $40 \pm 31,1$ & $7(24,1 \%)$ & Coliformi a $37^{\circ} \mathrm{C}$ & $0(0 \%)$ & - \\
\hline $27,5 \pm 9,6$ & $4(13,8 \%)$ & E. coli & $0(0 \%)$ & - \\
\hline- & $0(0 \%)$ & Enterococchi & $5(17,2 \%)$ & $64 \pm 64,2$ \\
\hline- & $0(0 \%)$ & P. aeruginosa & $0(0 \%)$ & - \\
\hline- & $0(0 \%)$ & C. perfringens & $0(0 \%)$ & - \\
\hline 20 & $1(3,4 \%)$ & S. aureus & $0(0 \%)$ & - \\
\hline - & $0(0 \%)$ & Aeromonas spp. & $0(0 \%)$ & - \\
\hline- & $0(0 \%)$ & Muffe & $16(55,1 \%)$ & $131,9 \pm 229,5$ \\
\hline Assente in $25 \mathrm{~g}$ & $0(0 \%)$ & L. monocytogenes & $0(0 \%)$ & Assente su $25 \mathrm{~mL}$ \\
\hline Assente in $25 \mathrm{~g}$ & $0(0 \%)$ & Salmonella spp. & $0(0 \%)$ & Assente su $25 \mathrm{~mL}$ \\
\hline
\end{tabular}

UFC, unità formanti colonie; CBT, carica batterica totale.

peratura media della bevanda cioccolato era pari a $67,6 \pm 4,2^{\circ} \mathrm{C}$ con valori variabili da 60 a $76^{\circ} \mathrm{C}$. Non si osservava nessun tipo di relazione tra CBT delle bevande e relativa temperatura di erogazione.

\section{Discussione}

Le analisi batteriologiche ottenute hanno confermato quanto macroscopicamente evidenziato nel corso del campionamento, circa le condizioni igieniche dei DA. Tra le maggiori criticità emerse ci sembra interessante sottolineare: i) le caratteristiche microbiologiche dell'acqua utilizzata, che si discostano nettamente da quanto previsto dal D. Lgs. $n^{\circ} 31$ del 02/02/2001 per le acque destinate al consumo umano (Repubblica Italiana, 2001), non possono che ripercuotersi negativamente sull'igiene complessiva dei DA; ii) l'evidente formazione di biofilm a livello del sistema di aspirazione e di circolazione dell'acqua; iii) l'estrema variabilità delle temperature dell'acqua di lavaggio, comprese tra $58,5^{\circ} \mathrm{C}$ e $80^{\circ} \mathrm{C}$ (media $70^{\circ} \mathrm{C}$ ), che non sembra essere in grado di ridurre la contaminazione batterica a livello del sistema di erogazione e miscelazione; iv) la non corretta gestione dei fondi di caffè che potrebbe aver favorito il frequente riscontro di muffe nella polvere di latte.

\section{Conclusioni}

Alla luce di quanto osservato, al di là dell'imprescindibile impiego di acqua rigorosamente potabile, le criticità sopra emerse possono essere facilmente risolte mediante l'applicazione di corrette good manufacturing practice (GMP). Il manuale di corretta prassi igienica dell'Associazione Italiana Distribuzione Automatica (Dragoni e Bonomi, 2007) prevede, infatti, la periodica pulizia con appositi detergenti sia del sistema di aspirazione e circolazione dell'acqua che di quello di erogazione e miscelazione, nonché una corretta gestione dei fondi di caffè. A tal riguardo è previsto, infatti, l'impiego di contenitori monouso (sacchetti) e la loro sostituzione ad ogni visita dell'operatore al fine di ridurre al minimo la diffusione delle muffe. Altro parametro da tenere in considerazione e da monitorare, vista l'estrema variabilità riscontrata, risulta essere la temperatura di erogazione delle bevande che rappresenta un indice dello stato di efficienza delle serpentine. Secondo quanto riportato da Dragoni e Bonomi (2007), l'erogazione del caffè espresso dovrebbe avvenire normalmente a temperature superiori $\mathrm{a}+80^{\circ} \mathrm{C}$, mentre quella delle bevande solubili a una temperatura superiore a $+70^{\circ} \mathrm{C}$. La temperatura di erogazione delle bevande al di sotto dei $65^{\circ} \mathrm{C}$, infatti, rappresenta il limite critico cui devono immediatamente far seguito le seguenti azioni correttive: pulizia, sanificazione e manutenzione straordinaria (Dragoni e Bonomi, 2007). La temperatura di $65^{\circ} \mathrm{C}$ garantirebbe, infatti, la potenziale assenza dei germi indicatori di contaminazione fecale (Hall, 2008), tuttavia da essa non si possono attendere effetti bonificanti se non a partire da contaminazioni molto basse, come chiaramente emerge dal nostro studio. 


\section{Bibliografia}

Commissione Europea, 2004. Regolamento del parlamento europeo e del consiglio del 29 aprile 2004 sull'igiene dei prodotti alimentari, 852/2004/CE. In: Gazzetta Ufficiale, L 139/1, 30/04/2004.

Dragoni I, Bonomi R, 2007. Manuale di corretta prassi igienica per la distribuzione automatica di alimenti. CONFIDA Associazione Italiana Distribuzione Automatica ed., Milano, Italia. Disponibile al sito: http://www.salute.gov.it/imgs/ C_17_pagine Aree_1187_listaFile_itemName_8_ file.pdf

Hall A, 2008. Microbiological risks associated with hot-drinks vending. Tesi di Dottorato, Università del Galles, UK. Disponibile al sito: http://repository.uwic.ac.uk/dspace/ bitstream/10369/860/1/Andrew\%20Hall\%20 -\%20PhD\%20thesis.pdf

Hunter PR, Burge SH, 1986. The microbiological quality of drinks from vending machines. J Hyg-Camb 97:497-500.
ISO, 1999. Microbiology of food and animal feeding stuffs - Horizontal method for the enumeration of coagulase-positive staphylococci (Staphylococcus aureus and other species) - Part 1: Technique using BairdParker agar medium Norma UNI EN ISO 6888-1:1999. Organizzazione internazionale per la normazione ed., Ginevra, Svizzera.

ISO, 2001. Microbiology of food and animal feeding stuffs. Horizontal method for the enumeration of beta-glucuronidase-positive Escherichia coli. Part 2: Colony-count technique at 44 degrees $\mathrm{C}$ using 5-bromo4-chloro-3-indolyl beta-D-glucuronide. Norma UNI EN ISO 16649-2:2001. Organizzazione internazionale per la normazione ed., Ginevra, Svizzera.

ISO, 2005. Microbiologia di alimenti e mangimi per animali: metodo orizzontale per la ricerca e la conta di Listeria monocytogenes. Parte 1: metodo per la ricerca. Norma UNI EN ISO 11290-1:2005. Organizzazione internazionale per la normazione ed., Ginevra, Svizzera.
ISO, 2006. Microbiology of food and animal feeding stuffs. Horizontal method for the enumeration of coliforms. Colony-count technique. Norma UNI EN ISO 4832:2006. Organizzazione internazionale per la normazione ed., Ginevra, Svizzera.

ISO, 2008. Metodo per rilevare la presenza di Salmonella spp. in alimenti per uso umano e mangimi. Norma UNI EN ISO 6579:2008. Organizzazione internazionale per la normazione ed., Ginevra, Svizzera.

Repubblica Italiana, 2001. Attuazione della direttiva no. 98/83/CE relativa alla qualità delle acque destinate al consumo umano, DL 31/2001. In: Gazzetta Ufficiale no. 52, 03/03/2001. Disponibile al sito: http//www. camera.it/parlam/leggi/deleghe/01031dl.htm

Vallone L, Bonomi R, 2011. Qualità igienicosanitaria di bevande erogate da distributori automatici. Ital J Food Safety 1:81-4.

White AS, Godard RD, Belling C, Kasza V, Beach R, 2010. Beverages obtained from soda fountain machines in the U.S. contain microorganisms, including coliform bacteria. Int J Food Microbiol 137:61-6. 